\title{
Magnetic Properties of Transition Metal Monolayers on Ta(001) Surfaces
}

\author{
S. J. Youn ${ }^{1 *}$ and S. C. Hong ${ }^{2}$ \\ ${ }^{1}$ Department of Physics Education and Research Institute of Natural Science, Gyeongsang National University, Jinju 660-701, Korea \\ ${ }^{2}$ Department of Physics, University of Ulsan, Ulsan 680-749, Korea
}

(Received 21 October 2008, Received in final form 25 November 2008, Accepted 25 November 2008)

\begin{abstract}
The magnetic and structural properties of transition metal (Mn, Fe, Co) monolayers on Ta(001) surfaces are investigated theoretically by using the first principles full-potential linearized augmented plane wave method. $\mathrm{Mn}$ and Fe monolayers become ferromagnetic on Ta(001) surfaces while Co monolayers becomes non-magnetic. The paramagnetism of Co monolayers is explained by the Stoner theory of magnetism. The magnetic coupling of a transition metal overlayer with a substrate is ascribed to the orbital hybridization between the $s$ and $d$ orbitals of the transition metal.
\end{abstract}

Keywords : transition metal, magnetism, surface magnetism, Ta

\section{Introduction}

When transition metals are put on a surface, they exhibit different forms of magnetic behavior depending on the conditions of the substrate. For example, an Mn monolayer becomes ferromagnetic on a W(001) surface while it is an antiferromagnet on noble metal surfaces. However, bulk $\mathrm{Mn}$ is a complex antiferromagnet. An Fe monolayer becomes an antiferromagnet on a W(001) surface while it is a ferromagnet on noble metal surfaces. $\mathrm{Fe}$ is a typical ferromagnet in bulk state. Co is also predicted to be an antiferromagnet on a W(001) surface and a ferromagnet on noble metal surfaces [2]. The atomic number of the transition metal plays an important role in determining the magnetic behavior, since the magnetic moment is determined on how the d-orbital is filled with electrons. For example, early transition metals ( $\mathrm{Cr}, \mathrm{Mn}$ ) are antiferromagnetic and late transition metals (Fe, Co, $\mathrm{Ni})$ are ferromagnetic on $\mathrm{Pd}(001)$. This trend is reversed on a $\mathrm{W}$ substrate [3].

The magnetic behavior of transition metals on $\mathrm{W}(001)$ surfaces has been studied in detail by the Blügel group [2, $3]$. It will be interesting to compare the magnetic properties when the substrates are changed to materials other than W. Ta is a good candidate as a substitute of W. Ta has the same bcc crystal structure as $\mathrm{W}$ with an enlarged

*Corresponding author: Tel: +82-55-751-5651

Fax: +82-55-763-4608, e-mail: ysj@gnu.kr lattice constant. Furthermore, Ta belongs to the same $5 d$ series as $\mathrm{W}$ with an atomic number that is smaller by one. We have investigated theoretically the magnetic properties of transition metal ( $\mathrm{Mn}, \mathrm{Fe}$, and $\mathrm{Co}$ ) monolayers grown on $\mathrm{Ta}(001)$ surfaces by using the first principle full-potential linearized augmented plane wave (FP-LAPW) method.

\section{Method}

The electronic structure was calculated using the FPLAPW $[4,5]$ method within a generalized gradient approximation [6]. The FP-LAPW method is an all electron calculation with no shape approximation for charge densities, potentials, and wave functions. The charge densities and potentials inside the muffin-tins are expanded by the lattice harmonics up to $l_{\max }=8$. Core electrons are treated fully relativistically and valence electrons are treated semirelativistically. Transition metal monolayers on $\mathrm{Ta}(001)$ surfaces were modeled by single slabs with 9 symmetrical layers in total. The experimental value of the lattice constant of $3.30 \AA$ for bulk Ta was adopted. The muffin-tin radii are 2.4 a.u. for Ta and 2.2 a.u. for $3 \mathrm{~d}-$ transition metals. The interlayer distances between the $\mathrm{Ta}(001)$ surfaces and the transition metal monolayers were determined by minimizing the total energies. The magnetic behavior of paramagnetic (PM), antiferromagnetic (AFM) and ferromagnetic (FM) states was considered. For magnetic calculations, a $\mathrm{p}(1 \times 1)$ structure was 
used for $\mathrm{PM}$ and FM and a $\mathrm{c}(2 \times 2)$ structure was used for AFM. We used $k_{\max }=3.0$ for $\mathrm{Fe} / \mathrm{Ta}(001)$ and $\mathrm{Co} / \mathrm{Ta}(001)$, which corresponds to about 1400 basis functions. For $\mathrm{Mn} / \mathrm{Ta}(001), k_{\max }=2.9$ was used, which corresponds to about 1300 basis functions. $21 k_{\|}$points in the irreducible wedge of the two dimensional Brillouin zone were used for integration in k-space in all the calculations.

\section{Results}

The total energies were calculated to obtain an equilibrium distance between the overlayer and the top layer in the PM, AFM, and FM states. Fig. 1 shows the total energy curves for $\mathrm{Fe} / \mathrm{Ta}(001)$ as a typical example. The PM states have the highest total energy while the FM states have the lowest total energy. The total energy of $\mathrm{Mn} / \mathrm{Ta}(001)$ shows a similar behavior as that of $\mathrm{Fe} / \mathrm{Ta}$ (001). Co atoms are expected to have a magnetic moment of about $1 \mu_{\mathrm{B}}$ on $\mathrm{Ta}(001)$ due to the tendency that the magnitude of the magnetic moment decreases as the atomic number increases from $\mathrm{Mn}$ to $\mathrm{Co}$. However, $\mathrm{Co} / \mathrm{Ta}(001)$ is nonmagnetic at its equilibrium distance. The paramagnetism of $\mathrm{Co} / \mathrm{Ta}(001)$ is explained below by the Stoner theory of magnetism. The results regarding structure and magnetic behavior are summarized in Table 1. The FM

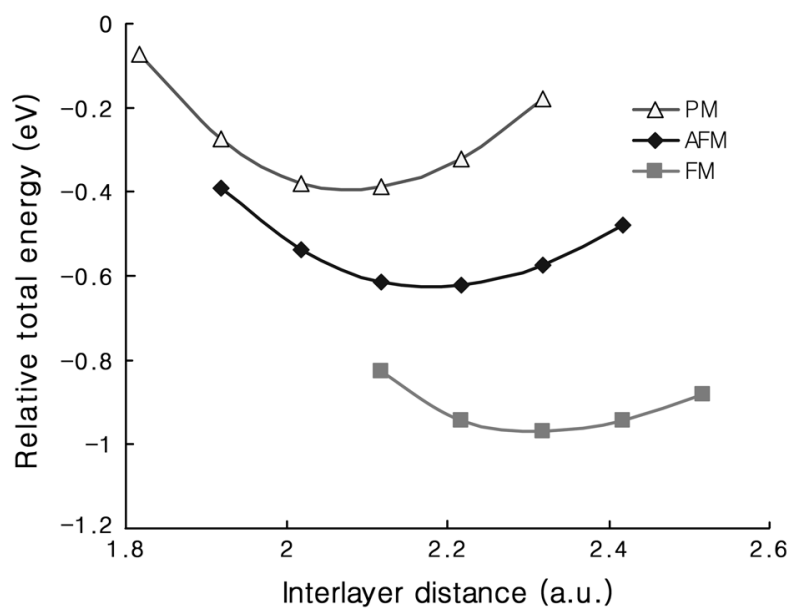

Fig. 1. Relative total energies of $\mathrm{Fe} / \mathrm{Ta}(001)$ in $\mathrm{PM}$, AFM, and FM states as a function of the interlayer distance. Zero energy has no significant meaning here but is the total energy for a paramagnetic state.

Table 1. Equilibrium properties.

\begin{tabular}{cccc}
\hline \hline & Magnetic state & $\begin{array}{c}\text { Magnetic moment } \\
\left(\mu_{\mathrm{B}}\right)\end{array}$ & $\begin{array}{c}\text { Overlayer distance } \\
(\AA)\end{array}$ \\
\hline $\mathrm{Mn}$ & $\mathrm{FM}$ & 3.1 & 2.66 \\
$\mathrm{Fe}$ & $\mathrm{FM}$ & 2.1 & 2.31 \\
$\mathrm{Co}$ & $\mathrm{PM}$ & 0.0 & 2.15 \\
\hline
\end{tabular}

states are stable for the $\mathrm{Mn}$ and $\mathrm{Fe}$ overlayers on a $\mathrm{Ta}(001)$ surface while the PM state is most stable for the Co overlayer. Kubetzka et al. also reported an FM ground state for $\mathrm{Fe} / \mathrm{Ta}(001)$ [1]. The magnetic moment of Fe, 2.1 $\mu_{\mathrm{B}}$, is similar to the bulk value of $2.2 \mu_{\mathrm{B}}$. This stands in contrast to the usual tendency that the magnetic moment increases on a surface [7]. It has been reported that the magnetic moment of $\mathrm{Fe}$ is reduced to $1.72 \mu_{\mathrm{B}}$ due to relaxation on a $\mathrm{Cr}$ (001) surface [8]. Among the overlayers, both the interlayer distance and the magnetic moment are largest for $\mathrm{Mn}$. The equilibrium PM overlayer distances are $2.18 \AA, 2.08 \AA$, and $2.15 \AA$ for $\mathrm{Mn}, \mathrm{Fe}$, and $\mathrm{Co}$, respectively. When magnetism is turned on, the overlayers distances are increased, which means that magnetic interactions are important in determining the overlayer distances.

For a better understanding of the paramagnetic behavior of $\mathrm{Co} / \mathrm{Ta}(001)$, the $d$-orbital local DOS (LDOS) of the overlayer $\mathrm{Co}$ atom as well as of Fe and $\mathrm{Mn}$ are plotted in Fig. 2 for the equilibrium overlayer distances. The change of overall shape of the LDOS as the atomic number changes can be explained with the rigid band model. As the atomic number increases from $\mathrm{Mn}$ to $\mathrm{Co}$, the energy band moves towards lower energy as the band is filled. There is a peak at the Fermi level in the LDOS of Mn while the Fermi level is located at the trough of the LDOS for Co. According to the Stoner theory of magnetism, a paramagnetic state is not stable if $I N(0)>1$ where $N(0)$ is the LDOS at the Fermi level and $I$ is the Stoner parameter of the transition metal overlayer atom. The Stoner parameters are $0.41 \mathrm{eV}, 0.46 \mathrm{eV}$, and $0.49 \mathrm{eV}$ for metallic $\mathrm{Mn}, \mathrm{Fe}$, and $\mathrm{Co}$, respectively [2]. Hence, the Stoner factors $[\operatorname{IN}(0)]$ are $1.62,1.32$, and 0.38 for the $\mathrm{Mn}$, $\mathrm{Fe}$, and $\mathrm{Co}$ overlayers, resepectively, which means that the PM state is stable for $\mathrm{Co} / \mathrm{Ta}(001)$ and unstable for $\mathrm{Mn} / \mathrm{Ta}(001)$ and $\mathrm{Fe} / \mathrm{Ta}(001)$. Although we have used the Stoner parameters of the bulk transition metals, the Stoner theory explains the paramagnetism of $\mathrm{Co} / \mathrm{Ta}(001)$ quali-

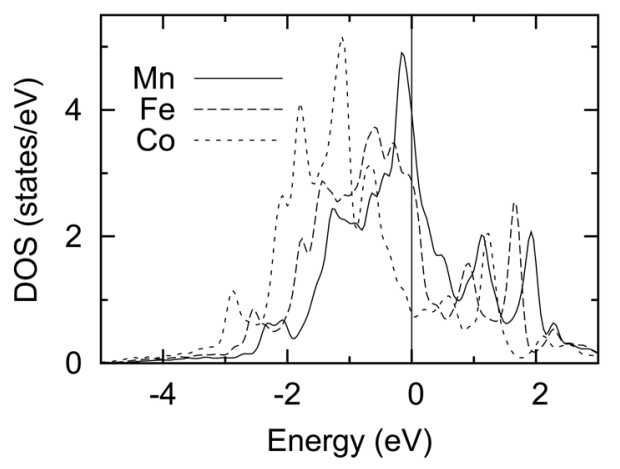

Fig. 2. $d$-orbital LDOS of the overlayers ( $\mathrm{Co}, \mathrm{Mn}$, and $\mathrm{Fe})$ at the equilibrium overlayer distance. 

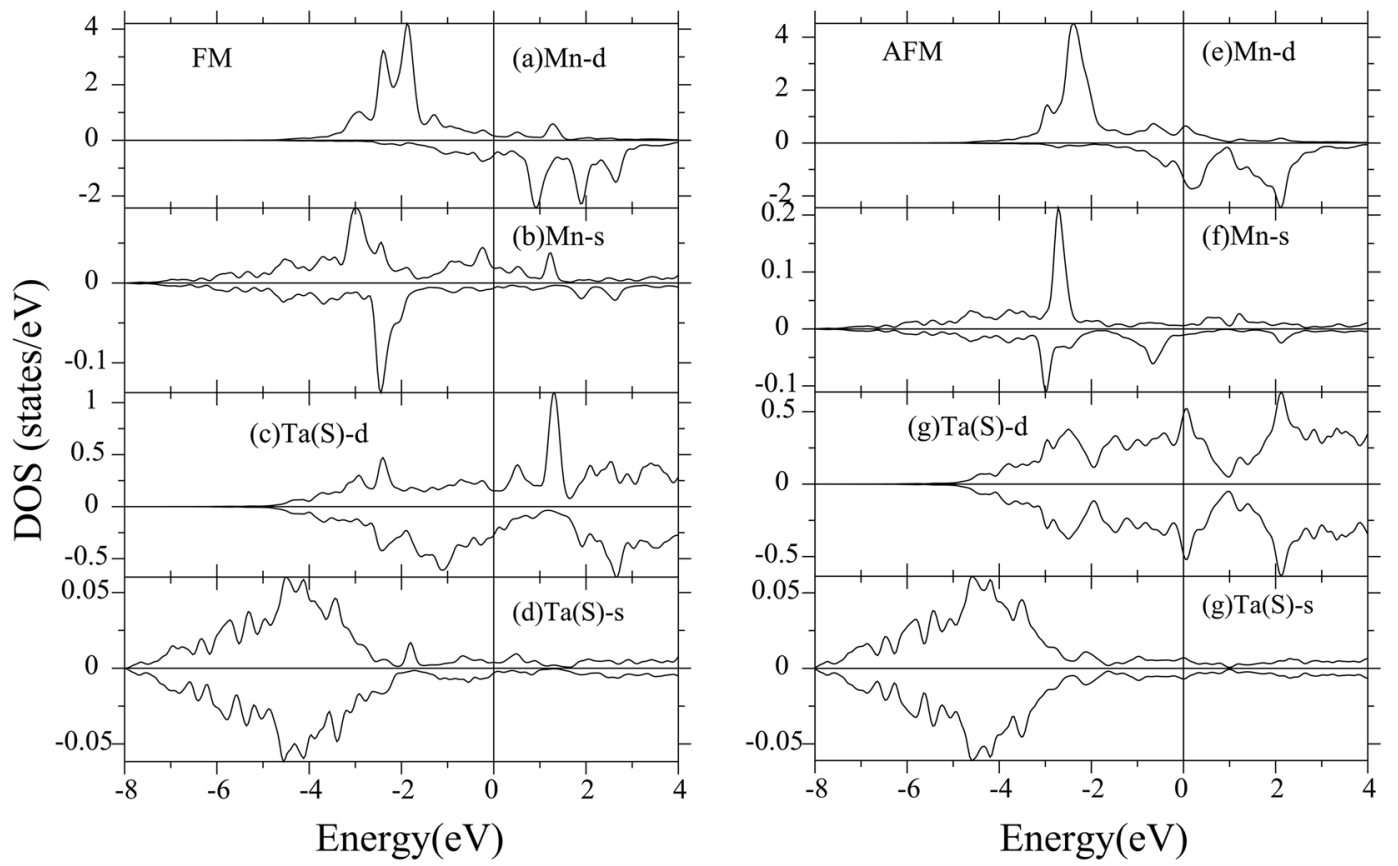

Fig. 3. LDOS of the overlayer Mn and top layer Ta at the equilibrium interlayer distance. Positive or negative sign of LDOS represents spin direction.

tatively.

In order to explain the ferromagnetic properties of $\mathrm{Mn}$ and Fe monolayers on $\mathrm{Ta}(001)$, we investigated the LDOS of the transition metal overlayers and the top layer Ta. For example, Fig. 3 shows the local DOSs of the Mn-s, Mn- $d$, Ta- $s$, and Ta- $d$ orbitals for the FM and AFM Mn/Ta(001).

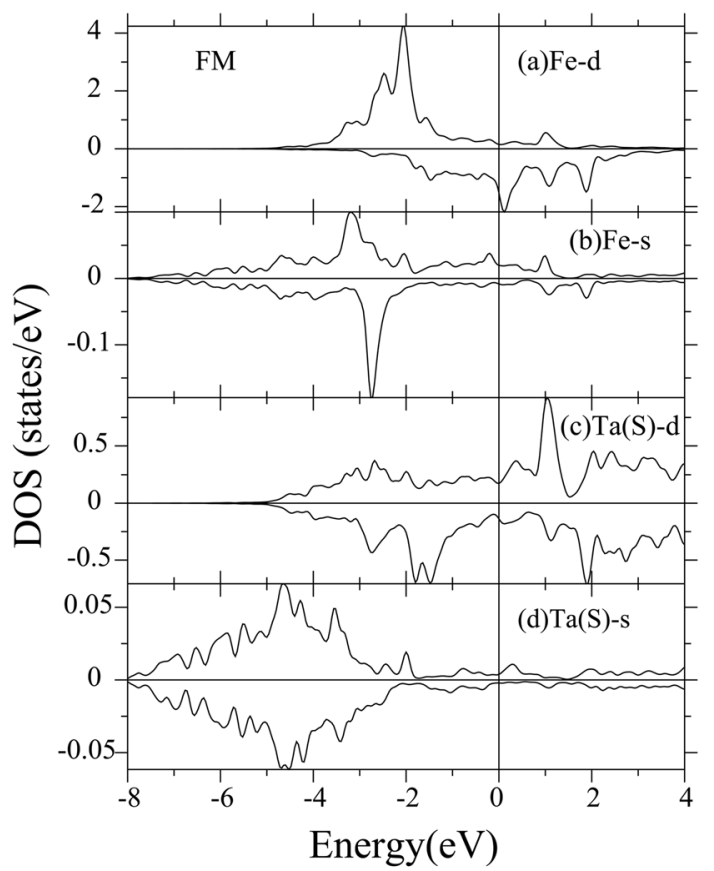

The Mn- $d_{\uparrow}$ band is almost fully occupied, while the $\mathrm{Mn}-d_{\downarrow}$ is partially occupied, which gives Mn a magnetic moment of $3.1 \mu_{\mathrm{B}}$. The magnetic moment in $\mathrm{Mn}$ atoms becomes stable through coupling with substrate atoms as well as other Mn atoms in the overlayer. Since the Mn-d electrons are localized in character, coupling occurs via

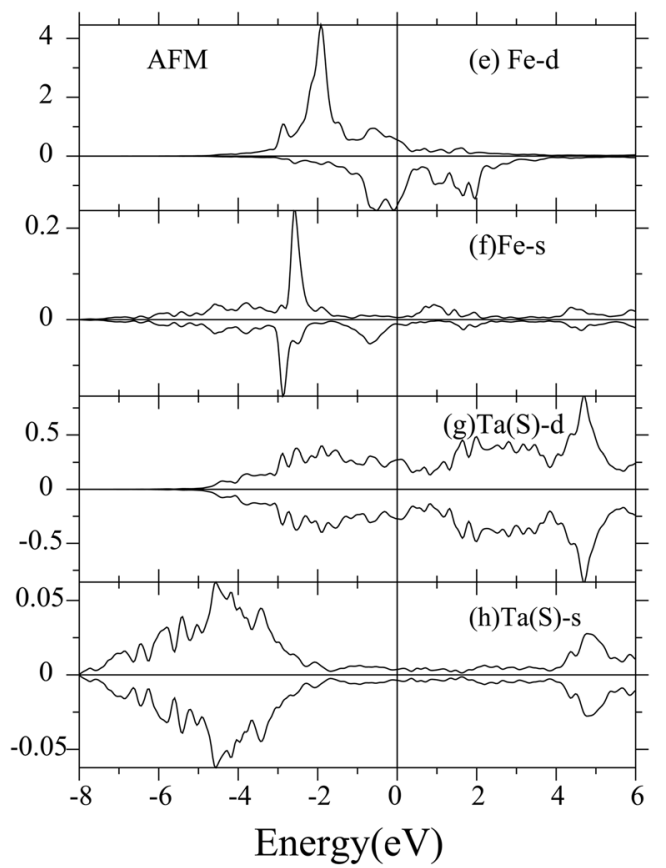

Fig. 4. LDOS of the overlayer Fe and top layer Ta at the equilibrium interlayer distance. Positive or negative sign of LDOS represents spin direction. 


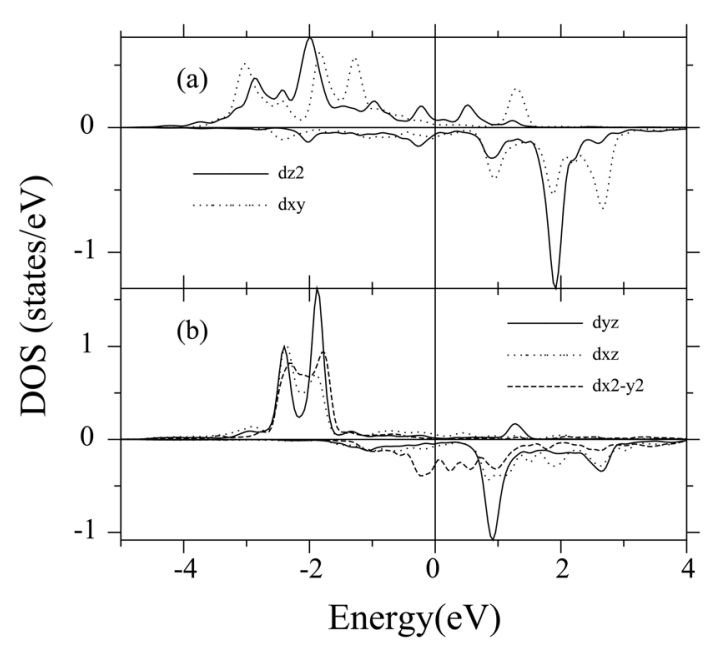

Fig. 5. LDOS split by the crystal field of overlayer $d$-orbitals at the equilibrium interlayer distance. (a) $d_{\mathrm{z}^{2}}$ (solid) and $d_{\mathrm{xy}}$ (dotted) (b) $d_{\mathrm{yz}}$ (solid), $d_{\mathrm{xz}}$ (dotted), and $d_{\mathrm{x}^{2}-\mathrm{y}^{2}}$ (dashed).

the overlayer $s$-orbitals. Considering that magnetic moment is determined by the Mn- $d \uparrow$ orbital, the $\uparrow$ electrons in the

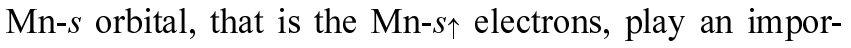

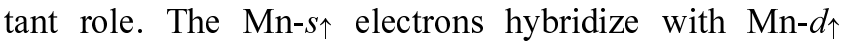
electrons at $-3.0 \mathrm{eV}$ below the Fermi level for FM as can be seen in Fig. 3(b), while this occurs at $-2.7 \mathrm{eV}$ for AFM as can be seen in Fig. 3(f). The lower total energy of the FM state than that of the AFM state can be ascribed to the location of Mn- $s \uparrow$ orbitals in the DOS. The Ta- $d$ electrons of the top layer are more important than the Ta-s electrons in coupling with Mn atoms as can be seen in Figs. 3(c) and (d). Fig. 4 depicts the local DOSs of the Fe-s, Fe- $d$, Ta-s, and Ta- $d$ orbitals for the FM and $\mathrm{AFM} \mathrm{Fe} / \mathrm{Ta}(001)$. The $\mathrm{Fe}-s \uparrow$ electronic states are located at $-3.2 \mathrm{eV}$ for FM and $-2.6 \mathrm{eV}$ for AFM, which reflects the lower total energy of FM than that of AFM.

In order to observe in detail the role of the Mn- $d$ orbital in hybridization, the LDOSs of Mn- $d$ orbitals were split according to the crystal field in Fig. 5 as a typical example. Note the DOS at $-3 \mathrm{eV}$, where the magnetic coupling is made, in which electronic states consist mainly of Mn- $d_{\mathrm{z}^{2}}$ and Mn- $d_{\mathrm{xy}}$ orbitals. From the shape of the orbitals, we can expect that Mn- $d_{\mathrm{z}^{2}}$ electrons are magnetically coupled with the substrate and that $\mathrm{Mn}-d_{\mathrm{xy}}$ electrons couple with adjacent $\mathrm{Mn}$ atoms in the overlayer. Electrons from $d_{\mathrm{yz}}, d_{\mathrm{xz}}$, and $d_{\mathrm{x}^{2}-\mathrm{y}^{2}}$ orbitals are most important in the forming of the magnetic moments in the overlayer.

\section{Conclusion}

We have investigated the structural and magnetic properties of transition metal monolayers on $\mathrm{Ta}(001)$ surfaces. $\mathrm{Fe}$ and $\mathrm{Mn}$ monolayers have ferromagnetic ground states while a Co monolayer has a paramagnetic ground state. The paramagnetic behavior of Co layers can be explained with the Stoner theory since the paramagnetic state has a low DOS at the Fermi level while those of Mn and Fe are high enough to give rise to magnetic orderings. Magnetic coupling of the transition metal overlayer occurs when the $s$ electrons of the transition metal hybridize with $d_{\mathrm{z}^{2}}$ and $d_{x y}$ orbitals in the same atom. From the shape of the orbitals, we see that the $d_{\mathrm{z}^{2}}$ electrons are to couple with the substrate and that the $d_{\mathrm{xy}}$ electrons are to couple with other adjacent transition metals in the overlayer.

\section{Acknowledgments}

This work was supported by KOSEF (Grant Nos. R012007-000-11593-0 and R0A-2006-000-1024).

\section{References}

[1] A. Kubetzka, et al., Phys. Rev. Lett. 94, 087204 (2005).

[2] P. Ferriani, S. Heinze, G. Bihlmayer, and S. Blügel, Phys. Rev. B 72, 024452 (2005).

[3] J. F. Janak, Phys. Rev. B 16, 255 (1977).

[4] E. Wimmer, H. Krakauer, M. Weinert, and A. J. Freeman, Phys. Rev. B 24, 864 (1981).

[5] M. Weinert, E. Wimmer, and A. J. Freeman, Phys. Rev. B 26, 4571 (1982).

[6] J. P. Perdew and Y. Wang, Phys. Rev. B 45, 13244 (1992).

[7] Y. J. Jin and J. I. Lee, J. Magnetics 12(2), 59 (2007).

[8] I. G. Kim, J. I. Lee, Y. R. Jang, and S. C. Hong, J. Magnetics 1(1), 9 (1996). 